\title{
Mittag-Leffler Operator Connected with Certain Subclasses of Bazilevič Functions
}

\author{
Om Ahuja $\mathbb{D}^{1},{ }^{1}$ Asena Çetinkaya $\mathbb{D}^{2},{ }^{2}$ and Naveen Kumar Jain $\mathbb{D}^{3}$ \\ ${ }^{1}$ Department of Mathematical Sciences, Kent State University, Ohio 44021, USA \\ ${ }^{2}$ Department of Mathematics and Computer Science, İstanbul Kültür University, Istanbul, Turkey \\ ${ }^{3}$ Department of Mathematics, Aryabhatta College, Delhi 110021, India
}

Correspondence should be addressed to Om Ahuja; oahuja@kent.edu

Received 24 July 2021; Accepted 27 December 2021; Published 19 January 2022

Academic Editor: Gaetano Luciano

Copyright (c) 2022 Om Ahuja et al. This is an open access article distributed under the Creative Commons Attribution License, which permits unrestricted use, distribution, and reproduction in any medium, provided the original work is properly cited.

In this paper, we introduce a new generalized class of analytic functions involving the Mittag-Leffler operator and Bazilevic functions. We examine inclusion properties, radius problems, and an application of the generalized Bernardi-Libera-Livingston integral operator for this function class.

\section{Introduction}

Let $\mathscr{A}$ be the family of all functions of the form

$$
f(z)=z+\sum_{n=2}^{\infty} a_{n} z^{n},
$$

which are analytic in the open unit disc $\mathbb{D}:=\{z:|z|<1\}$. Denote by $\delta$ the subfamily of $\mathscr{A}$ consisting of functions that are univalent in $\mathbb{D}$. Let $\mathscr{K}, \mathcal{S}^{*}$, and $\mathscr{C}$ be the well-known subclasses of $\mathcal{S}$ consisting of functions that are, respectively, convex, starlike (with respect to the origin), and close-toconvex in $\mathbb{D}$. The class $\mathscr{P}$ consists of analytic functions that are analytic in $\mathbb{D}$ and satisfies the conditions $p(0)=1$ and
$\operatorname{Re} p(z)>0$ in $\mathbb{D}$ and is also well-known in the theory of univalent functions. For definitions, properties, and history of these classes, one may refer to a survey article by the first author [1, 2]. Recently, Ali et al. [3] and Anand et al. [4] studied these classes to find various radius problems.

The Mittag-Leffler function $E_{\alpha}$, defined by

$$
E_{\alpha}(z)=\sum_{n=0}^{\infty} \frac{z^{n}}{\Gamma(\alpha n+1)}, \quad(\alpha \in \mathbb{C} ; \operatorname{Re}(\alpha)>0 ; z \in \mathbb{C}),
$$

was introduced in 1903 by Mittag-Leffler [5, 6] in connection with his method of summation of some divergent series. A general form of this special function (2) given by

$$
E_{\alpha, \beta}(z)=\sum_{n=0}^{\infty} \frac{z^{n}}{\Gamma(\alpha n+\beta)}, \quad(\alpha, \beta \in \mathbb{C} ; \operatorname{Re}(\alpha)>0 ; \operatorname{Re}(\beta)>0 ; z \in \mathbb{C}),
$$

which was studied by Wiman [7] in 1905. During these last twenty-five years, interest in Mittag-Leffler type functions (2) and (3) has significantly increased among engineers and scientists due to their applications in numerous applied problems, such as fluid flow, diffusive transport skin to diffusion, electric networks, probability, and statistical distribution theory. For detailed account of various properties and references related to applications, one may refer to $[8,9]$. 
Motivated by Sivastava and Tomovski [10], Attiya [11] studied certain applications of the generalized Mittag-Leffler operator involving differential subordination.

Corresponding to the function $E_{\alpha, \beta}$, Elhaddad et al. [12] introduced the Mittag-Leffler linear operator $\mathscr{E}_{\lambda, \alpha, \beta}^{m} f: \mathscr{A} \longrightarrow \mathscr{A}$ given by

$$
\mathscr{E}_{\lambda, \alpha, \beta}^{m} f(z)=z+\sum_{n=2}^{\infty} \frac{\Gamma(\beta)(1+(n-1) \lambda)^{m}}{\Gamma(\alpha(n-1)+\beta)} a_{n} z^{n},
$$

where $m \in \mathbb{N}_{0}=\mathbb{N} \bigcup\{0\}, \lambda \geq 0, \alpha, \beta \in \mathbb{C}, \operatorname{Re}(\alpha)>0, \operatorname{Re}(\beta)>0$ and when $\operatorname{Re}(\alpha)=0$ and $\beta \neq 0$. From (4), the following recurrence formula can be easily obtained:

$$
\mathscr{E}_{\lambda, \alpha, \beta}^{m+1} f(z)=(1-\lambda) \mathscr{E}_{\lambda, \alpha, \beta}^{m} f(z)+\lambda z\left(\mathscr{E}_{\lambda, \alpha, \beta}^{m} f(z)\right)^{\prime},
$$

where $m \in \mathbb{N}_{0}$ and $\lambda \geq 0$. For suitable values of the parameters $m, \alpha, \beta$, and $\lambda$, we may get several linear operators; for example,

(1) For $\alpha=0$ and $\beta=1$, we get Al-Oboudi operator [13].

(2) For $\alpha=0, \beta=1$, and $\lambda=1$, we get Sălăgean operator [14].
(3) For $m=0$ and $\lambda=1$, we get the operator

$$
\mathbb{E}_{\alpha, \beta}(z)=z \Gamma(\beta) E_{\alpha, \beta}(z)=z+\sum_{n=2}^{\infty} \frac{\Gamma(\beta)}{\Gamma(\alpha(n-1)+\beta)} z^{n}
$$

Let us denote by $\mathscr{B}(\vartheta, \tau, g, p)$ or briefly denote by $\mathscr{B}$, a class of functions $f \in \mathscr{A}$ for which $p \in \mathscr{P}, g \in \mathcal{S}^{*}$, and real numbers $\vartheta, \tau$ with $\vartheta>0$ such that

$$
f(z)=\left[(\vartheta+i \tau) \int_{0}^{z} p(t) g(t)^{\vartheta} t^{i \tau-1} \mathrm{~d} t\right]^{1 /(\vartheta+i \tau)},
$$

where powers are taken as principal values. Bazilevich [15] proved that $\mathscr{B} \subset \mathcal{S}$. In fact, it is known that $\mathscr{K} \subset \mathcal{S}^{*} \subset \mathscr{C} \subset \mathscr{B} \subset \mathcal{S}$. For $\vartheta>0$ and $\rho<1$, Ponnusamy and Karunakaran [16] showed that

$\mathscr{B}_{1}(\vartheta, \rho)=\left\{f \in \mathscr{A}: \operatorname{Re}\left(\frac{z f^{\prime}(z)}{f^{1-\vartheta}(z) g^{\vartheta}(z)}\right)>\rho, z \in \mathbb{D}\right\}$.

For $g(z) \equiv z$, these authors observed that

$$
\begin{aligned}
& \mathscr{B}_{2}(\vartheta, \rho)=\left\{f \in \mathscr{A}: \operatorname{Re}\left(\frac{z f^{\prime}(z)}{f(z)}\left(\frac{f(z)}{z}\right)^{\vartheta}\right)>\rho, z \in \mathbb{D}\right\}, \\
& \mathscr{B}_{3}(\rho):=\mathscr{B}_{2}(0, \rho)=\left\{f \in \mathscr{A}: \operatorname{Re}\left(\frac{z f^{\prime}(z)}{f(z)}\right)>\rho, z \in \mathbb{D}\right\}=\mathcal{S}^{*}(\rho), \\
& \mathscr{B}_{4}(\rho):=\mathscr{B}_{2}(1, \rho)=\left\{f \in \mathscr{A}: \operatorname{Re}\left(f^{\prime}(z)\right)>\rho, z \in \mathbb{D}\right\}=\mathscr{P}^{\prime}(\rho) .
\end{aligned}
$$

In view of (7), Singh [17] observed that $\mathscr{B}_{1}(\vartheta, 0)$, $\mathscr{B}_{2}(\vartheta, 0), \mathscr{B}_{3}(0)=\mathcal{S}^{*}$, and $\mathscr{B}_{4}(0)=\mathscr{P}$ are subclasses of $\mathscr{B}$. For further details, one may refer to $[15,18]$.

In 1976, Padmanabhan and Parvatham [19] introduced the class $\mathscr{P}_{k}(\rho)$ of analytic functions $p$ defined in $\mathbb{D}$ satisfying the properties $p(0)=1$ and

$$
\int_{0}^{2 \pi}\left|\frac{\operatorname{Re} p(z)-\rho}{1-\rho}\right| \mathrm{d} \theta \leq k \pi
$$

where $z=r e^{i \theta}, k \geq 2$, and $0 \leq \rho<1$. In fact, he proved the following important result.

Lemma 1 (see [19]). If $p \in \mathscr{P}_{k}(\rho)$, then

$$
p(z)=\frac{1}{2} \int_{0}^{2 \pi} \frac{1+(1-2 \rho) z e^{-i \theta}}{1-z e^{-i \theta}} \mathrm{d} \mu(\theta)
$$

where $\mu(\theta)$ is a function with bounded variation on $[0,2 \pi]$ such that

$$
\begin{gathered}
\int_{0}^{2 \pi} \mathrm{d} \mu(\theta)=2 \pi \\
\int_{0}^{2 \pi}|\mathrm{d} \mu(\theta)| \leq k \pi .
\end{gathered}
$$

From (10), it is observed that $p \in \mathscr{P}_{k}(\rho)$ if and only if there exists $p_{1}, p_{2} \in \mathscr{P}(\rho)$ such that (see [18])

$$
p(z)=\left(\frac{k}{4}+\frac{1}{2}\right) p_{1}(z)-\left(\frac{k}{4}-\frac{1}{2}\right) p_{2}(z), \quad(z \in \mathbb{D}) .
$$

We note that, for $\rho=0$, we obtain the class $\mathscr{P}_{k}(0):=\mathscr{P}_{k}$ defined by Pinchuk [20]. For $k=2$, we get $\mathscr{P}_{2}(\rho):=\mathscr{P}(\rho)$ the class of analytic functions with positive real part greater than $\rho$, and for $k=2$ and $\rho=0$, we have the class $\mathscr{P}_{2}(0):=\mathscr{P}$ of functions with positive real part.

Motivated by many researchers in $[5-7,10-12,15$, $17,18,21]$, we introduce the following generalized class of Mittag-Leffler-Bazilevic operator involving the class $\mathscr{P}_{k}(\rho)$. 
Definition 1. Let $k \geq 2,0 \leq \rho<1, \vartheta>0, m \in \mathbb{N}_{0}, \lambda \geq 0$, and $\gamma \in \mathbb{C}$ be such that $\operatorname{Re}(\gamma)>0$. Then, a function $f \in \mathscr{A}$ given by (1) is in the class $\mathscr{M}_{\lambda, \alpha, \beta}^{m, \gamma}(k, \vartheta, \rho)$ if it satisfies the condition

$$
\left\{(1-\gamma)\left(\frac{\mathscr{E}_{\lambda, \alpha, \beta}^{m} f(z)}{z}\right)^{\vartheta}+\gamma\left(\frac{\mathscr{E}_{\lambda, \alpha, \beta}^{m+1} f(z)}{z}\right)\left(\frac{\mathscr{E}_{\lambda, \alpha, \beta}^{m} f(z)}{z}\right)^{\vartheta-1}\right\} \in \mathscr{P}_{k}(\rho)
$$

where $z \in \mathbb{D}$.

This new class $\mathscr{M}_{\lambda, \alpha, \beta}^{m, \gamma}(k, \vartheta, \rho)$ involves several subclasses of the family $\mathscr{B}$ of Bazilevic functions defined by (7); see [15]. For example, $\mathscr{M}_{1,0,1}^{0,1}(2, \vartheta, 0)=\mathscr{B}_{2}(\vartheta, 0)$, $\mathscr{M}_{1,0,1}^{0,1}(2,0,0)=\mathscr{B}_{3}(0)$, and $\mathscr{M}_{1,0,1}^{0,1}(2,1,0)=\mathscr{B}_{4}(0)$ are subclasses of $\mathscr{B}$; see $[15,17]$. In fact, for different values of $m, \vartheta, \lambda, \alpha, \beta, \gamma, k$ and $\rho$, the class $\mathscr{M}_{\lambda, \alpha, \beta}^{m, \gamma}(k, \vartheta, \rho)$ reduces to many important subclasses studied by various researchers. For instance,

(i) Setting $m=0, \lambda=1, \alpha=0$, and $\beta=1$, we get (see [18])

$$
\begin{aligned}
\mathscr{M}_{1,0,1}^{0, \gamma}(k, \vartheta, \rho) & =: \mathscr{M}^{\gamma}(k, \vartheta, \rho) \\
& =\left\{f \in \mathscr{A}:(1-\gamma)\left(\frac{f(z)}{z}\right)^{\vartheta}+\gamma \frac{z f^{\prime}(z)}{f(z)}\left(\frac{f(z)}{z}\right)^{\vartheta} \in \mathscr{P}_{k}(\rho), 0 \leq \rho<1\right\} .
\end{aligned}
$$

(ii) Setting $m=0, \lambda=1, \alpha=0, \gamma=1, \beta=1$, and $k=2$, we get (see [15])

$$
\mathscr{M}_{1,0,1}^{0,1}(2, \vartheta, \rho)=: \mathscr{B}_{2}(\vartheta, \rho)=\left\{f \in \mathscr{A}: \frac{z f^{\prime}(z)}{f(z)}\left(\frac{f(z)}{z}\right)^{\vartheta} \in \mathscr{P}(\rho), 0 \leq \rho<1\right\} .
$$

(iii) Setting $m=0, \lambda=1, \alpha=0, \beta=1, \gamma=0, \vartheta=1$, and $k=2$, we get (see [21])

$\mathscr{M}_{1,0,1}^{0,0}(2,1, \rho)=: \mathscr{M}(\rho)=\left\{f \in \mathscr{A}: \frac{f(z)}{z} \in \mathscr{P}(\rho), 0 \leq \rho<1\right\}$.

In view of the above examples, we conclude that the notion of generalized class $\mathscr{M}_{\lambda, \alpha, \beta}^{m, \gamma}(k, \vartheta, \rho)$ unifies several known subclasses of $\mathscr{A}$.

In this paper, we study various properties of the class $\mathscr{M}_{\lambda, \alpha, \beta}^{m, \gamma}(k, \vartheta, \rho)$. In particular, we investigate inclusion properties, radius problem, and an application of the generalized Bernardi-Libera-Livingston integral operator for this function class.

We list some preliminary lemmas required for proving our main results.

Lemma 2 (see [22]). Let $u=u_{1}+i u_{2}$ and $v=v_{1}+i v_{2}$, and suppose $\Psi(u, v)$ is a complex function satisfying the following conditions:

(i) $\Psi(u, v)$ is continuous in a domain $D \subset \mathbb{C}^{2}$ (ii) $(1,0) \in D$ and $\operatorname{Re} \Psi(1,0)>0$

(iii) $\operatorname{Re} \Psi\left(i u_{2}, v_{1}\right) \leq 0$, whenever $\left(i u_{2}, v_{1}\right) \in D$ and $v_{1} \leq-\left(\left(1+u_{2}^{2}\right) / 2\right)$

If $p(z)=1+c_{1} z+c_{2} z^{2}+\ldots$ is an analytic function in $\mathbb{D}$ such that $\left(p(z), z p^{\prime}(z)\right) \in D$ and $\operatorname{Re} \Psi\left(p(z), z p^{\prime}(z)\right)>0$ for $z \in \mathbb{D}$, then $\operatorname{Re} p(z)>0$ in $\mathbb{D}$.

Lemma 3 (see [23]). If $p$ is in $\mathscr{P}$, and if $\zeta$ is a complex number satisfying $\operatorname{Re}(\zeta) \geq 0, \zeta \neq 0$, then $\operatorname{Re}\left\{p(z)+\zeta z p^{\prime}(z)\right\}$ $>\rho(0 \leq \rho<1)$ implies that

$$
\operatorname{Re} p(z)>\rho+(1-\rho)\left(2 \iota_{1}-1\right)
$$

where

$$
\iota_{1}=\int_{0}^{1}\left(1+t^{\operatorname{Re}(\zeta)}\right)^{-1} \mathrm{~d} t
$$

$\iota_{1}$ is an increasing function of $\operatorname{Re}(\zeta)$ and $1 / 2 \leq \iota_{1}<1$. This estimate cannot be improved in general.

\section{Inclusion Properties}

In this section, we examine some inclusion properties for the class $\mathscr{M}_{\lambda, \alpha, \beta}^{m, \gamma}(k, \vartheta, \rho)$. 
Theorem 1. Let $\gamma>0$ and $f \in \mathscr{M}_{\lambda, \alpha, \beta}^{m, \gamma}(k, \vartheta, \rho)$. Then,

$$
\left(\frac{\mathscr{E}_{\lambda, \alpha, \beta}^{m} f(z)}{z}\right)^{\vartheta} \in \mathscr{P}_{k}\left(\rho_{1}\right) \text {, }
$$

where $\rho_{1}$ is given by

$$
\rho_{1}=\frac{2 \vartheta \rho+\lambda \gamma}{2 \vartheta+\lambda \gamma} .
$$

$$
\left(\frac{\mathscr{E}_{\lambda, \alpha, \beta}^{m} f(z)}{z}\right)^{\vartheta}=\left(1-\rho_{1}\right) p(z)+\rho_{1},
$$

where $p(0)=1$ and

$$
p(z)=\left(\frac{k}{4}+\frac{1}{2}\right) p_{1}(z)-\left(\frac{k}{4}-\frac{1}{2}\right) p_{2}(z)
$$

Thus, by using (14) and (22), we obtain

Proof. In view of (20), let

$\left\{(1-\gamma)\left(\frac{\mathscr{E}_{\lambda, \alpha, \beta}^{m} f(z)}{z}\right)^{\vartheta}+\gamma\left(\frac{\mathscr{E}_{\lambda, \alpha, \beta}^{m+1} f(z)}{z}\right)\left(\frac{\mathscr{E}_{\lambda, \alpha, \beta}^{m} f(z)}{z}\right)^{\vartheta-1}\right\}=(1-\gamma)\left(\left(1-\rho_{1}\right) p(z)+\rho_{1}\right)+\gamma\left[\frac{\mathscr{E}_{\lambda, \alpha, \beta}^{m+1} f(z)}{\mathscr{E}_{\lambda, \alpha, \beta}^{m} f(z)}\left(\left(1-\rho_{1}\right) p(z)+\rho_{1}\right)\right]$

Taking logarithmic differentiation of (22), we get

$$
\vartheta\left[\frac{z\left(\mathscr{E}_{\lambda, \alpha, \beta}^{m} f(z)\right)^{\prime}}{\mathscr{E}_{\lambda, \alpha, \beta}^{m} f(z)}-1\right]=\frac{\left(1-\rho_{1}\right) z p^{\prime}(z)}{\left(1-\rho_{1}\right) p(z)+\rho_{1}} .
$$

$$
\frac{\mathscr{E}_{\lambda, \alpha, \beta}^{m+1} f(z)}{\mathscr{E}_{\lambda, \alpha, \beta}^{m} f(z)}=\frac{\lambda\left(1-\rho_{1}\right) z p(z)}{\vartheta\left(\left(1-\rho_{1}\right) p(z)+\rho_{1}\right)}+1
$$

By using the identity (5) in the last expression, we obtain

$$
\begin{aligned}
\left(1-\rho_{1}\right) p(z)+\rho_{1}+\frac{\lambda \gamma\left(1-\rho_{1}\right) z p^{\prime}(z)}{\vartheta}= & \left(\frac{k}{4}+\frac{1}{2}\right)\left\{\left(1-\rho_{1}\right) p_{1}(z)+\rho_{1}+\frac{\lambda \gamma\left(1-\rho_{1}\right) z p_{1}(z)}{\vartheta}\right\} \\
& -\left(\frac{k}{4}-\frac{1}{2}\right)\left\{\left(1-\rho_{1}\right) p_{2}(z)+\rho_{1}+\frac{\lambda \gamma\left(1-\rho_{1}\right) z p_{2}(z)}{\vartheta}\right\} .
\end{aligned}
$$

Since $f \in \mathscr{M}_{\lambda, \alpha, \beta}^{m, \gamma}(k, \vartheta, \rho)$, it follows that

$$
\left\{\left(1-\rho_{1}\right) p_{i}(z)+\rho_{1}+\frac{\lambda \gamma\left(1-\rho_{1}\right) z p_{i}^{\prime}(z)}{\vartheta}\right\} \in \mathscr{P}(\rho), \quad(0 \leq \rho<1, i=1,2) .
$$

That is,

$$
\operatorname{Re} \Psi\left(i u_{2}, v_{1}\right)=\rho_{1}-\rho+\operatorname{Re}\left\{\frac{\lambda \gamma\left(1-\rho_{1}\right) v_{1}}{\vartheta}\right\}
$$

$$
\frac{1}{1-\rho}\left\{\left(1-\rho_{1}\right) p_{i}(z)+\rho_{1}+\frac{\lambda \gamma\left(1-\rho_{1}\right) z p_{i}^{\prime}(z)}{\vartheta}-\rho\right\} \in \mathscr{P} .
$$

To prove the theorem, we will show that $p_{i} \in \mathscr{P}(i=1,2)$. We form the functional $\Psi(u, v)$ by taking $u=u_{1}+i u_{2}$ and $v=v_{1}+i v_{2}$ such that

$$
\Psi(u, v)=\left(1-\rho_{1}\right) u+\rho_{1}-\rho+\frac{\lambda \gamma\left(1-\rho_{1}\right) v}{\vartheta} .
$$

Using (29), it is easy to show that the first two conditions of Lemma 2 are satisfied. To verify condition (iii), we obtain

$$
\begin{aligned}
& \leq \rho_{1}-\rho-\frac{\lambda \gamma\left(1-\rho_{1}\right)\left(1+u_{2}^{2}\right)}{2 \vartheta} \\
& =\frac{2 \vartheta \rho_{1}-2 \vartheta \rho-\lambda \gamma\left(1-\rho_{1}\right)\left(1+u_{2}^{2}\right)}{2 \vartheta} \\
& =\frac{2 \vartheta\left(\rho_{1}-\rho\right)-\lambda \gamma\left(1-\rho_{1}\right)-\lambda \gamma\left(1-\rho_{1}\right) u_{2}^{2}}{2 \vartheta} \\
& :=\frac{A+B u_{2}^{2}}{2 C},
\end{aligned}
$$


where $v_{1} \leq-\left(\left(1+u_{2}^{2}\right) / 2\right)$. Now, $\operatorname{Re} \Psi\left(i u_{2}, v_{1}\right) \leq 0$ if

$$
\begin{aligned}
& A=2 \vartheta\left(\rho_{1}-\rho\right)-\lambda \gamma\left(1-\rho_{1}\right) \leq 0, \\
& B=-\lambda \gamma\left(1-\rho_{1}\right) \leq 0, \\
& C=\vartheta>0 .
\end{aligned}
$$

It follows from $A \leq 0, \rho_{1}$ given by (21) and $B \leq 0$ that $0 \leq \rho_{1}<1$. In view of Lemma 2 , for $p(z)=p_{i}(z)$, $p_{i} \in \mathscr{P}(i=1,2)$, we get $p \in \mathscr{P}_{k}\left(\rho_{1}\right)$. This proves the result.

For $m=0, \lambda=1, \alpha=0$, and $\beta=1$, Theorem 1 reduces to the following new result.

Corollary 1. Let $\gamma>0$ and $f \in \mathscr{M}^{\gamma}(k, \vartheta, \rho)$. Then, $(f(z) / z)^{\vartheta} \in \mathscr{P}_{k}\left(\rho_{1}\right)$, where $\rho_{1}$ is given by

$$
\rho_{1}=\frac{2 \vartheta \rho+\gamma}{2 \vartheta+\gamma}
$$

By using the inclusion relation given in Theorem 1, we prove the following result.

Theorem 2. Let $\vartheta>0$ and $0 \leq \gamma_{1}<\gamma_{2}$. Then, $\mathscr{M}_{\lambda, \alpha, \beta}^{m, \gamma_{2}}(k, \vartheta, \rho) \subset \mathscr{M}_{\lambda, \alpha, \beta}^{m, \gamma_{1}}(k, \vartheta, \rho)$.

Proof. Let $f \in \mathscr{M}_{\lambda, \alpha, \beta}^{m, \gamma_{2}}(k, \vartheta, \rho)$. Then, we have

$$
H_{2}(z)=\left\{\left(1-\gamma_{2}\right)\left(\frac{\mathscr{E}_{\lambda, \alpha, \beta}^{m} f(z)}{z}\right)^{9}+\gamma_{2}\left(\frac{\mathscr{E}_{\lambda, \alpha, \beta}^{m+1} f(z)}{z}\right)\left(\frac{\mathscr{E}_{\lambda, \alpha, \beta}^{m} f(z)}{z}\right)^{9-1}\right\} \in \mathscr{P}_{k}(\rho)
$$

In view of Theorem 1 , we conclude that

$$
\left(\frac{\mathscr{E}_{\lambda, \alpha, \beta}^{m} f(z)}{z}\right)^{\vartheta}:=H_{1}(z) \in \mathscr{P}_{k}\left(\rho_{1}\right) \subset \mathscr{P}_{k}(\rho)
$$

$$
\left\{\left(1-\gamma_{1}\right)\left(\frac{\mathscr{E}_{\lambda, \alpha, \beta}^{m} f(z)}{z}\right)^{\vartheta}+\gamma_{1}\left(\frac{\mathscr{E}_{\lambda, \alpha, \beta}^{m+1} f(z)}{z}\right)\left(\frac{\mathscr{E}_{\lambda, \alpha, \beta}^{m} f(z)}{z}\right)^{9-1}\right\}=\left(1-\frac{\gamma_{1}}{\gamma_{2}}\right) H_{1}(z)+\frac{\gamma_{1}}{\gamma_{2}} H_{2}(z)
$$

Because the class $\mathscr{P}_{k}(\rho)$ is a convex set (see [18]), it follows that the right side of (36) belongs to $\mathscr{P}_{k}(\rho)$, and therefore $f \in \mathscr{M}_{\lambda, \alpha, \beta}^{m, \gamma_{1}}(k, \vartheta, \rho)$.

For $m=0, \lambda=1, \alpha=0$, and $\beta=1$, Theorem 2 reduces to the following inclusion result.

Remark 1 (see [18]). Let $\vartheta>0$ and $0 \leq \gamma_{1}<\gamma_{2}$. Then, $\mathscr{M}^{\gamma_{2}}(k, \vartheta, \rho) \subset \mathscr{M}^{\gamma_{1}}(k, \vartheta, \rho)$.

\section{Radius Problem}

In this section, we examine certain radius problems.

Theorem 3. If a function $f \in \mathscr{A}$ satisfies
Thus, for $\gamma_{1} \geq 0$, we have

$$
\left(\frac{\mathscr{E}_{\lambda, \alpha, \beta}^{m} f(z)}{z}\right)^{\vartheta} \in \mathscr{P}_{k}(\rho),
$$

then $f \in \mathscr{M}_{\lambda, \alpha, \beta}^{m, \gamma}(k, \vartheta, \rho)$ for $|z|<r_{1}$, where

$$
r_{1}=\frac{\lambda \gamma+\vartheta-\sqrt{\lambda^{2} \gamma^{2}+2 \lambda \gamma \vartheta}}{\vartheta} .
$$

Proof. In view of (37), we have

$$
\left(\frac{\mathscr{E}_{\lambda, \alpha, \beta}^{m} f(z)}{z}\right)^{\vartheta}=(1-\rho) p(z)+\rho,
$$

where $p \in \mathscr{P}_{k}$. Hence, by using (5) and (39), we easily get

$$
\begin{aligned}
& \frac{1}{1-\rho}\left\{(1-\gamma)\left(\frac{\mathscr{E}_{\lambda, \alpha, \beta}^{m} f(z)}{z}\right)^{\vartheta}+\gamma\left(\frac{\mathscr{E}_{\lambda, \alpha, \beta}^{m+1} f(z)}{z}\right)\left(\frac{\mathscr{E}_{\lambda, \alpha, \beta}^{m} f(z)}{z}\right)^{\vartheta-1}-\rho\right\}=p(z)+\frac{\lambda \gamma z p(z)}{\vartheta} \\
= & \left(\frac{k}{4}+\frac{1}{2}\right)\left\{p_{1}(z)+\frac{\lambda \gamma z p_{1}(z)}{\vartheta}\right\}-\left(\frac{k}{4}-\frac{1}{2}\right)\left\{p_{2}(z)+\frac{\lambda \gamma z p_{2}(z)}{\vartheta}\right\},
\end{aligned}
$$

where $p_{1}, p_{2} \in \mathscr{P}$ and $z \in \mathbb{D}$.

Now, by using well-known estimates (see [2]) for the class $\mathscr{P}$ given by 


$$
\begin{aligned}
& \left|z p_{i}^{\prime}(z)\right| \leq \frac{2 r \operatorname{Re} p_{i}(z)}{(1-r)^{2}}, \\
& \operatorname{Re} p_{i}(z) \geq \frac{1-r}{1+r}, \quad(|z|<r<1 ; i=1,2 ; z \in \mathbb{D}),
\end{aligned}
$$

we have

$$
\begin{aligned}
\operatorname{Re}\left\{p_{i}(z)+\frac{\lambda \gamma z p_{i}(z)}{\vartheta}\right\} & \geq \operatorname{Re}\left\{p_{i}(z)-\frac{\lambda \gamma\left|z p_{i}^{\prime}(z)\right|}{\vartheta}\right\} \\
& \geq \operatorname{Re} p_{i}(z)\left\{1-\frac{2 \lambda \gamma r}{\vartheta(1-r)^{2}}\right\} \\
& =\operatorname{Re} p_{i}(z)\left\{\frac{\vartheta(1-r)^{2}-2 \lambda \gamma r}{\vartheta(1-r)^{2}}\right\} .
\end{aligned}
$$

The right hand side of the last inequality is positive if $|z|=r<r_{1}$, where $r_{1}$ is given by (38).

Letting $m=0, \lambda=1, \alpha=0$, and $\beta=1$, Theorem 3 reduces to the following new result.

Corollary 2. If a function $f \in \mathscr{A}$ satisfies $(f(z) / z)^{\vartheta} \in \mathscr{P}_{k}(\rho)$, then $f \in \mathscr{M}^{\gamma}(k, \vartheta, \rho)$ for $|z|<r_{2}$, where

$$
r_{2}=\frac{\gamma+\vartheta-\sqrt{\gamma^{2}+2 \gamma \vartheta}}{\vartheta} \text {. }
$$

Remark 2. If $m=\alpha=0, \gamma=\beta=\lambda=\vartheta=1$, and $k=2$ in Theorem 3, then $f$ is in $\mathscr{M}_{1,0,1}^{0,1}(2,1, \rho):=\mathscr{B}_{2}^{1}(1,1,1, \rho, 1,0)$ for $|z|<2-\sqrt{3} \approx 0.2679$. This result was proved in Theorem 3.4 in [24].

\section{Application of an Integral Operator}

In this section, we consider an application of the generalized Mittag-Leffler operator given by (4) involving the generalized Bernardi-Libera-Livingston integral operator $\mathscr{L}_{\sigma}: \mathscr{A} \longrightarrow \mathscr{A}$ given by

$$
\mathscr{L}_{\sigma} f(z)=\frac{\sigma+1}{z^{\sigma}} \int_{0}^{z} t^{\sigma-1} f(t) \mathrm{d} t, \quad(\sigma>-1) .
$$

From this operator, we easily get

$$
z\left(\mathscr{E}_{\lambda, \alpha, \beta}^{m} \mathscr{L}_{\sigma} f(z)\right)^{\prime}=(\sigma+1) \mathscr{E}_{\lambda, \alpha, \beta}^{m} f(z)-\sigma \mathscr{E}_{\lambda, \alpha, \beta}^{m} \mathscr{L}_{\sigma} f(z)
$$

For several special cases of this operator and related operators, one may refer to a survey article by the first two authors [25] and related references therein.

Theorem 4. Let $f \in \mathscr{A}$ and $\mathscr{L}_{\sigma} f$ be given by (44). If

$$
\left\{(1-\gamma) \frac{\mathscr{E}_{\lambda, \alpha, \beta}^{m} \mathscr{L}_{\sigma} f(z)}{z}+\gamma \frac{\mathscr{E}_{\lambda, \alpha, \beta}^{m} f(z)}{z}\right\} \in \mathscr{P}_{k}(\rho),
$$

then

$$
\frac{\mathscr{E}_{\lambda, \alpha, \beta}^{m} \mathscr{L}_{\sigma} f(z)}{z} \in \mathscr{P}_{k}(\iota), \quad(z \in \mathbb{D}),
$$

where $\iota$ is given by

$$
\iota=\rho+(1-\rho)\left(2 \iota_{1}-1\right)
$$

$$
\iota_{1}=\int_{0}^{1}\left(1+\frac{\gamma}{\sigma+1}\right)^{-1} \mathrm{~d} t
$$

Proof. Consider the function

$$
\frac{\mathscr{E}_{\lambda, \alpha, \beta}^{m} \mathscr{L}_{\sigma} f(z)}{z}=p(z)=\left\{\left(\frac{k}{4}+\frac{1}{2}\right) p_{1}(z)-\left(\frac{k}{4}-\frac{1}{2}\right) p_{2}(z)\right\} .
$$

Differentiating both sides and using (45), we get

$$
\frac{\mathscr{E}_{\lambda, \alpha, \beta}^{m} f(z)}{z}=p(z)+\frac{z p^{\prime}(z)}{\sigma+1} .
$$

If we use the identity given by (46), we obtain

$(1-\gamma) \frac{\mathscr{E}_{\lambda, \alpha, \beta}^{m} \mathscr{L}_{\sigma} f(z)}{z}+\gamma \frac{\mathscr{E}_{\lambda, \alpha, \beta}^{m} f(z)}{z}=p(z)+\frac{\gamma z p^{\prime}(z)}{\sigma+1} \in \mathscr{P}_{k}(\rho)$.

This implies that

$$
\operatorname{Re}\left\{p_{i}(z)+\frac{\gamma z p_{i}(z)}{\sigma+1}\right\}>\rho, \quad(i=1,2) .
$$

By using Lemma 3, we see that $\operatorname{Re}\left\{p_{i}(z)\right\}>\iota$, where $\iota$ is given by (48). Thus, we arrive at $p \in \mathscr{P}_{k}(\iota)$. This completes the proof.

Setting $m=0, \lambda=1, \alpha=0$, and $\beta=1$ in operator $\mathscr{E}_{\lambda, \alpha, \beta}^{m} f$, Theorem 4 gives the following result.

Corollary 3. Let $f \in \mathscr{A}$ and $\mathscr{L}_{\sigma} f$ be given by (44). If

$$
\left\{(1-\gamma) \frac{\mathscr{L}_{\sigma} f(z)}{z}+\gamma \frac{f(z)}{z}\right\} \in \mathscr{P}_{k}(\rho),
$$

then $\left(\mathscr{L}_{\sigma} f(z)\right) / z \in \mathscr{P}_{k}(\iota)$, where $\iota$ is given by

$$
\begin{aligned}
& \iota=\rho+(1-\rho)\left(2 \iota_{1}-1\right), \\
& \iota_{1}=\int_{0}^{1}\left(1+\frac{\gamma}{\sigma \sigma+1}\right)^{-1} \mathrm{~d} t .
\end{aligned}
$$

\section{Conclusion}

We conclude our investigation by remarking that the defined new generalized class of analytic functions involving the Mittag-Leffler operator and Bazilevic functions gives various well-known subclasses of Bazilevic functions as particular cases which in turn yields many known results as corollaries. 


\section{Data Availability}

No data were used to support this study.

\section{Disclosure}

A preprint of an earlier version is available at https://arxiv. org/abs/2109.13509.

\section{Conflicts of Interest}

The authors declare that they have no conflicts of interest.

\section{References}

[1] O. P. Ahuja, "The Bieberbach conjecture and its impact on the developments in geometric function theory," Mathematical Chronicle, vol. 15, pp. 1-28, 1986.

[2] A. W. Goodman, Univalent Functions, Mariner Publishing Company, FL, USA, 1983.

[3] R. M. Ali, N. K. Jain, and V. Ravichandran, "Bohr radius for classes of analytic functions," Results in Mathematics, vol. 74, no. 4, p. 13, 2019.

[4] S. Anand, N. K. Jain, and S. Kumar, "Sharp Bohr radius constants for certain analytic functions," Bulletin of the Malaysian Mathematical Sciences Society, vol. 44, no. 3, pp. 1771-1785, 2021.

[5] G. M. Mittag-Leffler, "Sur la nouvelle fonction $E_{\alpha}(x)$," Comptes Rendus de l'Academie des Sciences, Serie II, vol. 137, pp. 554-558, 1903.

[6] G. M. Mittag-Leffler, "Une generalisation de l'integrale de Laplace-Abel," Comptes Rendus de l'Academie des Sciences, Serie II, vol. 137, pp. 537-539, 1903.

[7] A. Wiman, "Über den Fundamentalsatz in der Teorie der Funktionen Ea(x)," Acta Mathematica, vol. 29, pp. 191-201, 1905.

[8] R. Gorenflo, A. A. Kilbas, F. Mainardi, and S. V. Rogosin, Mittag-Leffler Functions, Related Topics and Applications, Springer-Verlag, Berlin, Germany, 2014.

[9] H. J. Haubold, A. M. Mathai, and R. K. Saxena, "Mittag-Lefer functions and their applications," Journal of Applied Mathematics, vol. 2011, Article ID 298628, 51 pages, 2011.

[10] H. M. Srivastava and Ž. Tomovski, "Fractional calculus with an integral operator containing a generalized Mittag-Leffler function in the kernel," Applied Mathematics and Computation, vol. 211, no. 1, pp. 198-210, 2009.

[11] A. Attiya, "Some applications of Mittag-Leffler function in the unit disk," Filomat, vol. 30, no. 7, pp. 2075-2081, 2016.

[12] S. Elhaddad, H. Aldweby, and M. Darus, "On certain subclasses of analytic functions involving differential operator," Jnãnãbha, vol. 48, pp. 55-64, 2018.

[13] F. M. Al-Oboudi, "On univalent functions defined by a generalized Sălăgean operator," International Journal of Mathematics and Mathematical Sciences, vol. 2004, no. 27, pp. 1429-1436, 2004.

[14] G. S. Salagean, "Subclasses of univalent functions," Complex Analysis-Fifth Romanian-Finnish Seminar: Lecture Notes in Mathematics, Springer-Verlag, vol. 1013, pp. 362-372, , Berlin, Germany, 1983.

[15] I. E. Bazilevich, "On a case of integrability in quadratures of the Loewner-Kufarev equation," Matematicheskii Sbornik. Novaya Seriya, vol. 37, no. 3, pp. 471-476, 1955, in Russian.
[16] S. Ponnussamy and V. Karunakaran, "Differential subordination and conformal mappings," Complex Variables, Theory and Applicattion, vol. 11, pp. 79-86, 1989.

[17] R. Singh, "On bazilevic functions," Proceedings of the American Mathematical Society, vol. 38, no. 2, pp. 261-271, 1973.

[18] K. I. Noor, "On certain classes of analytic functions," Journal of Inequalities in Pure and Applied Mathematics, vol. 7, no. 2, 5 pages, Article ID 49, 2006.

[19] K. S. Padmanabhan and R. Parvatham, "Properties of a class of functions with bounded boundary rotation," Annales Polonici Mathematici, vol. 31, no. 3, pp. 311-323, 1976.

[20] B. Pinchuk, "Functions of bounded boundary rotation," Israel Journal of Mathematics, vol. 10, no. 1, pp. 6-16, 1971.

[21] M. P. Chen, "On the regular functions satisfting $\operatorname{Re}\{f(z) /(z)\}$ $>\alpha$, Bulletin of the Institute of Mathematics, Academia Sinica, vol. 3, no. 1, pp. 65-70, 1975.

[22] S. Miller, "Differential inequalities and Carathéodory functions," Bulletin of the American Mathematical Society, vol. 81, no. 1, pp. 79-81, 1975.

[23] S. Ponnussamy, "Differential subordination and Bazilevic functions," Proceedings of the Indian Academy of Sciences Mathematical Sciences, vol. 105, no. 2, pp. 169-186, 1995

[24] K. I. Noor, S. Mustafa, and B. Malik, "On some classes of p-valent functions involving Carlson-Shaffer operator," Applied Mathematics and Computation, vol. 214, no. 2, pp. 336-341, 2009.

[25] O. P. Ahuja and A. Çetinkaya, "A Survey on the theory of integral and related operators in Geometric Function Theory," in Mathematical Analysis and Computing, vol. 344, pp. 635-652, ICMAC: Springer, Singapore, 2021. 